\title{
State of the art conference on weight management in VA: Policy and research recommendations for advancing behavioral interventions
}

\author{
Robin M. Masheb, $P h D^{1,2}$, Stephanie H. Chan, MPH ${ }^{3}$, Susan D. Raffa, $P h D^{3}$, \\ Ronald Ackermann, MD MPH', Laura J. Damschroder, MS, MPH', Paul A. Estabrooks, PhD', \\ Gina Evans-Hudnall, $P h D^{7}$, Neil C. Evans, MD ${ }^{8}$, Trina Histon, PhD ${ }^{9}$, Alyson J. Littman, PhD ${ }^{10}$, \\ Tannaz Moin, MD, MBA, MSHS 1,12, Karin M. Nelson, MD MSHS ${ }^{10,13}$, Sherry Pagoto, PhD ${ }^{14}$, \\ Nico P. Pronk, PhD ${ }^{15}$, Deborah F. Tate, PhD ${ }^{16}$, and Michael G. Goldstein, MD ${ }^{3}$
}

${ }^{1}$ Yale School of Medicine, New Haven, CT, USA; ${ }^{2}$ VA Connecticut Healthcare System, West Haven, CT, USA; ${ }^{3}$ VHA National Center for Health Promotion and Disease Prevention, Durham, NC, USA; ${ }^{N}$ Northwestern University Feinberg School of Medicine, Chicago, IL, USA; ${ }^{5}$ VA Ann Arbor Center for Clinical Management Research, Ann Arbor, MI, USA; 'University of Nebraska Medical Center, Omaha, NE, USA; ${ }^{7}$ Michael E. DeBakey VA Medical Center, Houston, TX, USA; ${ }^{8}$ VA Office of Connected Care, Washington, DC, USA; ${ }^{9}$ Kaiser Permanente Care Management Institute, Washington, DC, USA; 10VA Puget Sound Healthcare System, Tacoma, WA, USA; " VA Greater Los Angeles Healthcare System, Los Angeles, CA, USA; ${ }^{2}$ David Geffen School of Medicine at UCLA, Los Angeles, CA, USA; ${ }^{33}$ University of Washington, Seattle, WA, USA; ${ }^{14}$ University of Massachusetts Medical School, Worcester, MA, USA; ${ }^{15}$ HealthPartners Institute, Minneapolis, MN, USA; ${ }^{16}$ University of North Carolina Gillings School of Global Public Health, Chapel Hill, NC, USA.

This article summarizes outcomes of the behavioral interventions work group for the Veterans Health Administration (VHA) State of the Art Conference (SOTA) for Weight Management. Sixteen VHA and non-VHA subject matter experts, representing clinical care delivery, research, and policy arenas, participated. The work group reviewed current evidence of efficacy, effectiveness, and implementation of behavioral interventions for weight management, participated in phone- and online-based consensus processes, generated key questions to address gaps, and attended an in-person conference in March 2016. The work group agreed that there is strong evidence for efficacy and effectiveness of core behavioral intervention components and processes, but insufficient evidence to determine the comparative effectiveness of multiple cliniciandelivered weight management modalities, as well as technologies that may or may not supplement cliniciandelivered treatments. Effective strategies for implementation of weight management services in VHA were identified. The SOTA work group's foremost policy recommendations are to establish a system-wide culture for weight management and to identify a population-level health metric to measure the impact of weight management interventions that can be tracked and clearly communicated throughout VHA. The work group's top research recommendation is to determine how to deploy and scale the most effective behavioral weight management interventions for Veterans.

KEY WORDS: obesity; overweight; weight management; weight loss; veterans.

J Gen Intern Med 32(Suppl 1):S74-S8

DOI: $10.1007 / \mathrm{s} 11606-016-3965-y$

(c) Society of General Internal Medicine 2017

Electronic supplementary material The online version of this article (doi:10.1007/s11606-016-3965-y) contains supplementary material, which is available to authorized users.

Published online March 7, 2017

\section{INTRODUCTION}

Obesity is a leading cause of preventable death, is one of the most serious public health problems faced by our nation, ${ }^{1}$ and disproportionately affects Veterans. Seventy-seven percent of Veterans seeking healthcare through the Veterans Health Administration (VHA) are classified as overweight/obese ${ }^{2}$ compared to 68 percent of the general population. ${ }^{1}$ To help Veterans strive for a healthy weight, VHA's National Center for Health Promotion and Disease Prevention (NCP) developed the MOVE!@ Weight Management Program.

Launched nationally in 2006, MOVE! is VHA's evidencebased, weight management health promotion program. ${ }^{3}$ Guided by national policy, MOVE! is both a comprehensive lifestyle intervention and a population-based prevention program available at every VHA medical center. Although the program is designed to address excess weight in Veterans who are either overweight [body mass index $(\mathrm{BMI}) \geq 25$ ] with an obesityrelated condition or obese $(\mathrm{BMI} \geq 30)$, any VHA patient can attend. The evidence-based, comprehensive lifestyle intervention includes dietary, physical activity, and behavioral components, delivered in group or individual format. This intervention can be stand-alone or used in conjunction with bariatric surgery or weight loss medications; there currently is no systematic integration of these interventions. The populationbased approach includes high annual screening and referral to weight management services with almost $95 \%$ of patients screened and referred annually. ${ }^{4}$ Video-conferencing of MOVE! groups to outpatient clinics increases the reach of weight management services. Additionally, technology-based delivery methods such as home telehealth, telephone lifestyle 
coaching, and a MOVE! Coach mobile application have been developed to further increase the population reach and support in-person services.

Critical for VA is ensuring that best practices for policy and research are established for the MOVE! program, in part through on-going evaluation of a research literature on weight management that differs greatly from the Veteran population and VHA healthcare system. In terms of policy, VA is operating one of the largest healthcare system weight management programs in the country without the benefit of guidelines for how to run such a program informed by other comparable health systems. In terms of research, VHA has the ability to set its own research agenda that is centered on the specific needs of the Veteran population and is funded through VA's Health Services Research \& Development (HSR\&D).

To identify policy recommendations and research priorities regarding weight management for Veterans, VHA convened three weight management work groups, comprised of experts in behavioral, surgical, and pharmacological interventions, to participate in a State of the Art (SOTA) Conference. This article summarizes the outcomes of the behavioral interventions work group.

\section{METHODS}

\section{Participants}

The SOTA Conference co-chairs and behavioral work group co-leads (RMM and MGG) identified 16 behavioral intervention experts who participated in the SOTA and are co-authors. Ten individuals (RMM, SHC, SDR, LJD, GEH, NCE, AJL, $\mathrm{TM}, \mathrm{KMN}$, and MGG) are affiliated with VHA, and six individuals (RA, PAE, TH, SP, NPP and DFT) represent other institutions including healthcare delivery systems and academia. Additional demographic variables, academic characteristics, and experience of the experts are listed in Table 1.

\section{Identifying Six Key Question Areas}

The SOTA Planning Committee developed a comprehensive list of questions. Members of the Behavioral Interventions Work Group then met remotely, using an on-line platform called Think Tank, to collectively discuss and cull the highest priority questions for the SOTA. Think Tank provides a range of communications tools that enable participants to discuss, refine, prioritize, and, ultimately, vote on the questions to be addressed. A final list of six questions, divided into two categories - efficacy and implementation/effectiveness of behavioral interventions (see Table 2) - were discussed at the in-person conference.

\section{Participant Pre-Work}

Members of all three SOTA Conference work groups reviewed articles and guidelines for screening and management of obesity (see Online Appendix A). The behavioral work group co-leads selected additional articles, specifically

Table 1 Demographic Variables, Academic Characteristics, and Experience of SOTA Behavioral Work Group Participants $(\mathbf{N}=16)$

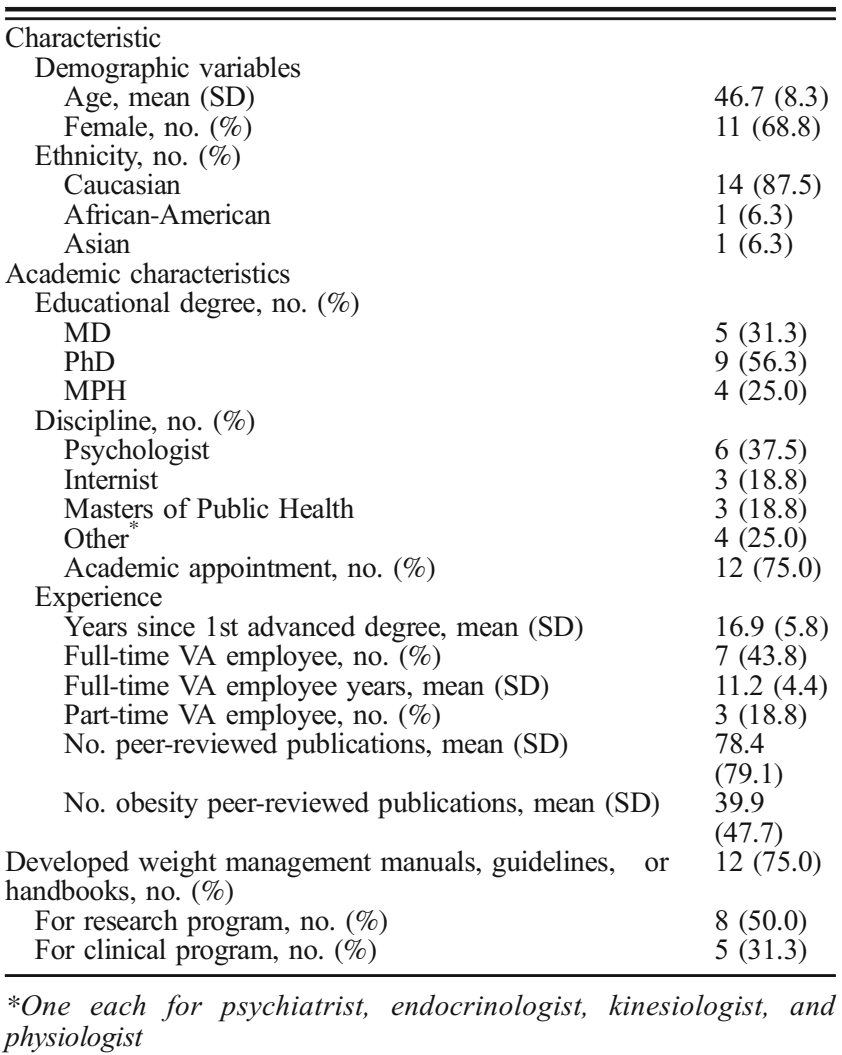

addressing the six key behavioral questions (see Online Appendix B). Articles were primarily limited to large-scale systematic reviews and meta-analyses or guidelines adhered to by multiple governmental or professional organizations. Work

Table 2 Six Key Question Areas for the Behavioral Interventions Work Group

\begin{tabular}{ll}
\hline \hline EFFICACY & EFFECTIVENESS/ \\
IMPLEMENTATION
\end{tabular}

1. Optimal Treatment Dose: What is the evidence that the effectiveness of behavioral interventions varies by the number of visits and length of treatment (the "dose" of behavioral intervention)? What is the evidence that there is a threshold effect (i.e., minimum dose) or a plateau effect?

2. Desirable Behavioral Interventions: What components or modalities (ways of delivering) of behavioral interventions have proven effective and should be emphasized for behavioral weight management?

3. Stepped-Care: What is the definition of "failure" to lose weight via behavioral weight management that would make a Veteran eligible/appropriate for weight loss medications or weight loss surgery?
1. Desirable Implementation Components: What is the most effective strategy (climate, facilitation roles, etc.) for implementing a standardized behavioral weight management, based on the lessons from the most effective sites?

2. Barriers \& Facilitators: What are the barriers and facilitators at the level of the patient, provider, facility, and health system to identification, referral, and sustained engagement of obese patients in behavioral weight management?

3. Reach: What is the effect of offering a less intensive (though still effective) intervention on reach and overall impact? How does reach $(\%$ of Veterans who utilize an intervention) vary by dose of intervention, e.g., minimal effective versus maximal effective dose? 
group members were asked to rank order the six questions by importance and to identify their level of expertise/ability to lead a discussion on each (see Online Appendix C).

\section{SOTA Conference}

The SOTA Conference began with a virtual Opening Plenary Session in January 2016 to convene all participants of the SOTA and provide a high-level overview of existing evidence related to weight management treatment in VHA. This was followed by individuals completing the aforementioned readings and culminated with a 2-day in-person meeting in March 2016. The work group co-leaders (RMM and MGG) and recorder (SHC) facilitated the 1st day of the in-person meeting. The day was divided into four sessions: (1) prioritization of key questions, (2) identification of effective components of behavioral interventions, (3) identification of effective strategies to improve implementation, and (4) identification of consensus among the work group members and prioritization of policy and research recommendations. A summary of these sessions was presented on the 2nd day of the in-person meeting to all SOTA participants.

\section{RESULTS}

\section{Consensus}

Sufficient evidence (SE) for comprehensive behavioral weight management treatment included: (1) three key intervention components (SE1), (2) program intensity (SE2), and (3) program structure (SE3). Work group members agreed there was sufficient evidence to indicate that efficacy of weight loss treatment is dependent upon three key intervention components: caloric restriction, physical activity promotion, and behavior modification. ${ }^{5,6}$ Systematic review of 12 trials with 3,893 participants deemed these components "necessary" to produce maximum weight loss for intensive, face-to-face comprehensive lifestyle intervention. ${ }^{7}$ Specifically, this includes advocating a strategy of moderately reduced calorie diet (deficit $\geq 500 \mathrm{kcal} /$ day), advocating a strategy of regular moderate-to-vigorous physical activity $(\geq 150 \mathrm{~min}$ per week or $\geq 30$ min most days of the week), and the use of behavioral strategies (self-monitoring, goal-setting with feedback, problem-solving, and reinforcement) to facilitate adherence to the diet and physical activity recommendations. Consensus was also reached for program intensity, determined by the number of sessions and length of treatment, and roughly categorized as low ( $<1$ session per month), moderate ( $1-2$ sessions per month over 6 to 12 months), and high ( $\geq 14$ sessions in 6 months). ${ }^{6}$ High-intensity programs generally produce greater weight loss than low-to-moderate programs. Moderate-to-high programs produce greater weight losses than usual care, and lowintensity programs do not produce outcomes superior to usual care. $^{6}$
Finally, there was agreement that sufficient evidence existed to describe the program structure necessary to obtain effective obesity treatment outcomes. ${ }^{8}$ Evidence directly came from VHA facilities offering a group-based intensive lifestyle intervention in which superior outcomes were obtained with a standard curriculum and with a defined treatment length and structure. ${ }^{9}$ Additional evidence came from the literature on health behavior counseling and patient engagement. Interventions such as the "5 A's" (Assess, Advise, Agree on goals, Assist in resolving barriers, and Arrange for follow-up) increase the likelihood of weight loss intervention effectiveness. ${ }^{10,11}$

Areas of insufficient evidence (IE) included: (1) comparative effectiveness of different weight loss intervention programming (IE1) and (2) strategies to increase the uptake, implementation and sustainability of weight management services (IE2). There was consensus that there is currently insufficient evidence to determine the comparative effectiveness of multiple clinician-delivered weight management modalities (group in-person, individual in-person, individual telephone, individual telehealth, and group video teleconferencing), as well as multiple technology-delivered weight management channels (text, email, website, mobile applications, and electronic weight check-ins) that may or may not be used as supplements to clinician-delivered modalities.

The work group also agreed that there was some limited, but insufficient evidence identifying specific strategies to increase the uptake, implementation, and sustainability of an effective weight management program. In VHA, high implementation facilities, in comparison to low implementation facilities, ${ }^{12}$ used networks and communication (interdisciplinary MOVE! teams that met regularly and communicated about MOVE! to other staff and patients), placed high priority on weight management compared to other initiatives, encouraged a learning climate (shared ideas with peers and superiors and regular forums to learn from others), and provided opportunities for reflection and evaluation (patient feedback prompting subsequent program changes such as the location of classes).

During the in-person meeting other topics, not covered in the literature, were discussed. Among these topics, the following areas were deemed essential for policy and/or research recommendations based upon expert opinion (EO): (1) development of a population indicator of impact (EO1), (2) the need to address integration of weight management services (behavioral, bariatric, and pharmacotherapy) (EO2), (3) treatment predictors (EO3), and (4) shared decision making (EO4). These areas are more fully described under the recommendations. See Tables 3 and 4 for alignment of evidence and recommendations.

\section{Policy Recommendations}

The work group developed several system-wide policy recommendations to increase the likelihood that evidence-based behavioral interventions are effectively implemented and sustained across VHA. First, it is critically important that highlevel leadership in VHA establish a vision and a culture that 
Table 3 Areas of Sufficient Evidence (SE), Insufficient Evidence (IE), and Expert Opinion (EO)

\begin{tabular}{lll}
\hline \hline $\begin{array}{l}\text { Sufficient } \\
\text { Evidence (SE) }\end{array}$ & $\begin{array}{l}\text { Insufficient Evidence } \\
\text { (IE) }\end{array}$ & $\begin{array}{l}\text { Expert Opinion } \\
\text { (EO) }\end{array}$ \\
\hline $\begin{array}{l}\text { SE1: Three } \\
\text { components of } \\
\text { behavioral }\end{array}$ & $\begin{array}{l}\text { IE1: Comparative } \\
\text { intervention } \\
\text { programming }\end{array}$ & $\begin{array}{l}\text { EO1: Population } \\
\text { indicator of } \\
\text { impact }\end{array}$ \\
$\begin{array}{l}\text { SE2: Program } \\
\text { intensity }\end{array}$ & $\begin{array}{l}\text { IE2: Strategies for } \\
\text { increasing uptake, } \\
\text { implementation, and } \\
\text { sustainability }\end{array}$ & $\begin{array}{l}\text { EO2: Weight } \\
\text { management } \\
\text { integration }\end{array}$ \\
$\begin{array}{l}\text { SE3: Program } \\
\text { structure }\end{array}$ & & $\begin{array}{l}\text { EO3: Treatment } \\
\text { predictors }\end{array}$ \\
& & $\begin{array}{l}\text { EO4: Shared } \\
\text { decision making }\end{array}$ \\
\hline
\end{tabular}

supports striving for a healthy weight through supportive directives (IE2) and clear policy statements about program structure (SE3). Second, VHA should develop a populationbased indicator of impact that combines intervention reach and effectiveness (EO1), for example, tracking the proportion of overweight/obese Veterans who achieve a meaningful weight loss (e.g., a 5\% weight loss to achieve a threshold for clinical risk reduction ${ }^{6}$ ). This metric could be used at multiple levels, including a provider's patient panel, a clinical population (e.g., diabetes), a facility or regional population, and the national VHA population. This metric could also be applied across behavioral, surgical, and pharmacological interventions to allow for consistent evaluation. Third, to support the implementation of behavioral interventions with program fidelity, VHA should establish and communicate core requirements of MOVE! to staff and coordinators (SE3), measure participation by intervention modality at multiple levels (facility, region, national) (IE1), and develop a system for tracking and giving feedback about Veteran engagement, program implementation, and fidelity (IE2 and EO1). Fourth, integration of weight management services that span behavioral, surgical, and

Table 4 VA Policy and Research Recommendations for Advancing Behavioral Interventions

\begin{tabular}{|c|c|c|c|}
\hline & Policy & & Research \\
\hline 1 & $\begin{array}{l}\text { VHA leadership vision } \\
\text { (IE2) and policies for pro- } \\
\text { gram structure (SE3) }\end{array}$ & 1 & $\begin{array}{l}\text { Comparative effectiveness of } \\
\text { different programming (IE1) }\end{array}$ \\
\hline 2 & $\begin{array}{l}\text { Population indicator of } \\
\text { impact (EO1) }\end{array}$ & 2 & $\begin{array}{l}\text { Strategies for increasing } \\
\text { uptake, implementation, and } \\
\text { sustainability (IE2) }\end{array}$ \\
\hline 3 & $\begin{array}{l}\text { Program fidelity } \\
\text { a. Establish and } \\
\text { communicate core } \\
\text { requirements (SE3) } \\
\text { b. Measure participation by } \\
\text { modality (EO1 \& IE1) } \\
\text { c. Veteran tracking \& } \\
\text { feedback (IE2) }\end{array}$ & 3 & $\begin{array}{l}\text { Factors/predictors associated } \\
\text { with weight loss (EO3) }\end{array}$ \\
\hline 4 & $\begin{array}{l}\text { Weight management } \\
\text { integration (EO2) }\end{array}$ & 4 & $\begin{array}{l}\text { Shared decision making } \\
\text { (EO4) } \\
\text { Data-driven quality } \\
\text { improvement (EO1) }\end{array}$ \\
\hline
\end{tabular}

Note: $S E=$ Sufficient evidence, $I E=$ insufficient evidence, and $E O=$ expert opinion pharmacological options should be established to adequately and appropriately distribute resources (EO2).

\section{Research Recommendations}

Behavioral work group members identified four key areas of needed research. First, assessment of the full range of weight management interventions in VHA, comparing outcomes across variations in intensity, modality, and technology assistance (IE1), is needed. Second, research is needed to develop and test effective interventions to improve the uptake, implementation, and sustainability of evidence-based behavioral weight loss practices (IE2). Third, we do not fully understand the patient characteristics that predict weight loss outcomes, or what factors might alter the course of outcome, such as treatment modality or patient preferences (EO3). In addition, shared decision making (SDM) is recommended for many types of healthcare decisions, but little is known about SDM in the context of weight management (EO4). Finally, there is a need for data-driven quality improvement for weight management to increase measurement impact, including research to improve the consistency of weight data entered into the electronic health record (EO1).

\section{Limitations}

Consensus and recommendations were drawn from research conducted with both civilian and Veteran populations; however, a recent review of the weight loss literature ${ }^{13}$ cautions that this literature has been primarily focused on young adult women. Thus, we have to consider the implications of generalizing findings from the broader weight loss literature to a Veteran population that is on average older and has a larger proportion of men. Findings from weight management trials of Veterans, however, may benefit civilians who are male, older, and have multiple medical and psychiatric morbidities and may generate greater interest in focusing on these patient groups outside VHA.

\section{Conclusions}

A work group consisting of VHA and non-VHA subject matter experts, representing clinical care delivery, research, and policy arenas, reached consensus regarding the key behavioral intervention components and processes required to achieve effective treatment outcomes. The work group's foremost policy recommendations are to establish a VHA vision that supports striving for a healthy weight for all Veterans and to identify a population-level health metric that evaluates the impact of behavioral weight management interventions (including behavioral, surgical, and pharmacological interventions) that can be tracked and clearly communicated throughout VHA. The work group's top research recommendation is to learn how VHA can reliably implement and scale the most effective behavioral weight management interventions for the benefit of Veterans. 
Acknowledgements: The authors would like to acknowledge David Atkins, MD, MPH, Matthew L. Maciejewski, PhD., and Geraldine McGlynn, MEd, for their support of the Behavioral Interventions Work Group and assistance with the SOTA.

Corresponding Author: Robin M. Masheb, PhD; VA Connecticut Healthcare System, 950 Campbell Ave Psychology Service, 116B. West Haven, CT 06516, USA (e-mail: robin.masheb@yale.edu).

\section{Compliance with ethical standards:}

Conflict of Interest: None of the authors acknowledge conflicts of interest with the exception of Deborah Tate, $\mathrm{PhD}$, who is on the Scientific Advisory Board for Weight Watchers.

Funding: The State of the Art Conference was supported by the Department of Veterans Affairs, Veterans Health Administration, Office of Research and Development, Health Services Research and Development.

Disclaimer: The views expressed in this article are those of the authors and do not necessarily reflect the position or policy of the Department of Veterans of Affairs or the US Government.

\section{REFERENCES}

1. Flegal KM, Kruszon-Moran D, Carroll MD, Fryar CD, Ogden CL. Trends in obesity among adults in the United States, 2005-2014. JAMA. 2016;315(21):2284-91.

2. Kahwati LC, Lance TX, Jones KR, Kinsinger LS. RE-AIM evaluation of the Veterans Health Administration's MOVE! Weight Management Program. Transl Behav Med. 2011;1(4):551-60.
3. MOVE! Weight Management Program. http://www.move.va.gov/. Accessed July 13, 2016.

4. Littman AJ, Damschroder LJ, Verchinina L, et al. National evaluation of obesity screening and treatment among veterans with and without mental health disorders. Gen Hosp Psychiatry. 2015;37(1):7-13.

5. Moyer VA, Force USPST. Screening for and management of obesity in adults: US Preventive Services Task Force recommendation statement. Ann Intern Med. 2012; 157(5):373-8.

6. Jensen MD, Ryan DH, Apovian CM, et al. 2013 AHA/ACC/TOS guideline for the management of overweight and obesity in adults: a report of the American College of Cardiology/American Heart Association Task Force on Practice Guidelines and The Obesity Society. J Am Coll Cardiol. 2014;63(25 Pt B):2985-3023.

7. Wadden TA, Butryn ML, Hong PS, Tsai AG. Behavioral treatment of obesity in patients encountered in primary care settings: a systematic review. JAMA. 2014;312(17):1779-91.

8. Kahwati L, Jacobs S, Kane H, Lewis M, Viswanathan M, Golin CE. Using qualitative comparative analysis in a systematic review of a complex intervention. Syst Rev. 2016;5(1):82.

9. Kahwati LC, Lewis MA, Kane H, et al. Best practices in the Veterans Health Administration's MOVE! Weight management program. Am J Prev Med. 2011;41(5):457-64.

10. Estabrooks PA, Glasgow RE, Dzewaltowski DA. Physical activity promotion through primary care. JAMA. 2003;289(22):2913-6.

11. Whitlock EP, Orleans CT, Pender N, Allan J. Evaluating primary care behavioral counseling interventions: an evidence-based approach. Am J Prev Med. 2002;22(4):267-84.

12. Damschroder LJ, Goodrich DE, Robinson CH, Fletcher CE, Lowery JC. A systematic exploration of differences in contextual factors related to implementing the MOVE! weight management program in VA: a mixed methods study. BMC Health Serv Res. 2011;11:248.

13. Gudzune KA, Doshi RS, Mehta AK, et al. Efficacy of commercial weightloss programs: an updated systematic review. Ann Intern Med. 2015;162:501-12. 